\title{
Pulley Injury in Climbing - A New Concept to Understand and Prevent It
}

\author{
Renato Carvalho Vilella* \\ Department of Autonomous Researcher, IMAM University, Brazil
}

*Corresponding author: Renato Carvalho Vilella, Department of Autonomous Researcher, IMAM University, Minas Gerais, Brazil

\begin{abstract}
The pulley injuries are not common among the average population, but when the subject is climbing (sports or rock, bouldering or leading) it gets clear that is common and lead the athlete to a path of serious consequences. With the evolution of the sport, the graduation level of difficulty in climbing will raise. Eventually the exposition of the climber to higher loads will rise also. This can be a factor for increased hand and finger injuries, specifically pulley injuries. The objective of this short review and new concept is to induce a new line of clinical reasoning to prevent pulley injuries when analyzing and treating athletes of climbing.
\end{abstract}

Keywords: Pulley injurie prevention; Sports climbing; Rock climbing; Surgery prevention

\section{Introduction}

The pulley injuries are not common among the average population, but when the subject is climbing (sports or rock, bouldering or leading) it gets clear that is common and lead the athlete to a path of serious consequences. There are not many articles in the scientific literature that exposes the pulley injuries in climbers. That is why there is no prevention guideline about it. With the evolution of the sport, the graduation level of difficulty in climbing will raise. Eventually the exposition of the climber to higher loads will rise also. This can be a factor for increased hand and finger injuries, specifically pulley injuries. The pulley act as supporters to the fingers tendons and helps to optimize the finger flexion, keeping the tendons close to the bones [1]. The pulleys are divided into 5 annular pulleys and 3 cruciform pulleys [1,2]. The pulleys A2 and A4 gets more attention at the studies because they are strictly related with the insertion of the flexor digitorum profundus (FDP) and with the flexor digitorum superficialis (FDS), respectively. With this relation, the loads on these pulleys are higher due the traction of the finger flexor tendons. The objective of this short review and new concept is to induce a new line of clinical reasoning when analyzing and treating athletes of climbing.

\section{The Climbing Grips}

There are two common grips described in literature when the subject is pulley injuries, the crimp grip and slope grip. In the crimp grip the proximal interphalangeal joint stays in flexion and the distal interphalangeal joint stays in hyperextension. In the slope grip both interphalangeal joints stay in flexion [3]. In 2006 Vigouroux et al. described the amount of force on pulleys and tendons that each grip generates. The crimp grip was able to generate 36 times more forces in crimp grip than in the slope grip. The electromyogram exam the FDP was the prime flexor at the crimp grip [3], but that is one way to interpret the values. The FDP showed more intensity of contraction activity, but that is explained by the fact of the distal interphalangeal joint being hyper-extended. The action of the FDP is flexion of the interphalangeal joint, if it is on passive insufficiency (hyperextension of the interphalangeal joint), the FDP will try harder to flex the distal interphalangeal joint. It shows how much strength the FDP does in the crimp grip, when the FDS does not make that much strength (it is not on passive insufficiency). So, the prime flexor at the crimp grip turns to be the FDS. 


\section{Outcomes of the Injury and Surgery}

The pulley injurie are the main injurie on the fingers in climbing athletes [4]. The time after full conservative rehabilitation to regain training level after complete pulley rupture was a mean of 8.8 months [3]. The time after surgery to gradual return on climbing was of 3 months [1]. This can take the athlete out of the competition and training, making the evolution of the athlete difficulty (time for rehabilitation) or impossible (depending of the injury level). In both cases it is a long-time off training and/or competing. This outcome has a significant role at the national and world context of sports and rock climbing. Imagine an elite athlete with a pulley injury on the eve of the Olympics, maybe it was the only opportunity that this athlete would have.

\section{What Have Been Missed?}

The two tendon flexors (superficial and deep) works together, they split the load on the finger and on the pulley. The pulleys A2 and A4 are strictly related to the insertion of the two tendons cited, respectively. The crimp grip can generate more force and that is why it is preferred among the climbers. The A2 pulley is the most common injury pulley [5]. The distal phalange of the finger stays on extension and this puts the FDP on insufficiency and increase the load on the FDS. Consequently, this increase the traction of the FDS tendon and as the pulley $\mathrm{A} 2$ has a function of supporting this tendon, the load at the A2 pulley raises also. With time, the most probably outcome is an injurie for overuse and stress at the pulley.

\section{Conclusion}

With this new concept of pulley injury mechanism, the solution is to prevent instead to treat it. The prevention can be made by training the FDP progressively [6]. When the hyper-extension occurs the FDS will not be so overloaded. The specifically training of the FDS is also recommended, creating a movement strategy with the capacity to absorb the force without being overloaded and consequently preventing the pulley injury. Other solution is to tape the distal interphalangeal joint at the neutral position, making impossible to this joint hyperextend. This can lead the athlete to conditioning the way to hold the grip and eventually the athlete will not need to use it anymore. More studies are necessary to develop strategies of movement coordination and prevention plans. Meanwhile, the solution presented on this article can be valuable for clinicians and coaches when evaluating a climbing athlete.

\section{References}

1. Schneeberger M, Schweizer A (2016) Pulley Ruptures in Rock Climbers: Outcome of Conservative Treatment with the Pulley-Protection Splint-A Series of 47 Cases. Wilderness \& Environmental Medicine 27(2): 211218.

2. Erik N Kubiak, Jeffrey A Klugman, Joseph A Bosco (2006) Hand Injuries in Rock Climbers. Bulletin of the NYU Hospital for Joint Diseases 64(34): 172-177.

3. Vigouroux L, Quaine F, Labarre Vila A, Moutet F (2006) Estimation of finger muscle tendon tensions and pulley forces during specific sportclimbing grip techniques. Journal of Biomechanics 39(14): 2583-2592.

4. Vilella R (2019) Sports Climbing Injury Prevention. Figshare 1: 6084.

5. Moutet F, Bouyer M, Corcella D, Forli A, Semere A (2019) Climber's Pulley Injuries. American Jewish Yearbook, pp. 81-99.

6. Bouyer M, Forli A, Semere A, Chedal Bornu BJ, Corcella D, et al. (2016) Recovery of rock-climbing performance after surgical reconstruction of finger pulleys. Journal of Hand Surgery 41(4): 406412.
This work is licensed under Creative Commons Attribution 4.0 License

To Submit Your Article Click Here:

Submit Article

DOI: $10.32474 /$ SCSOAJ.2019.02.000147

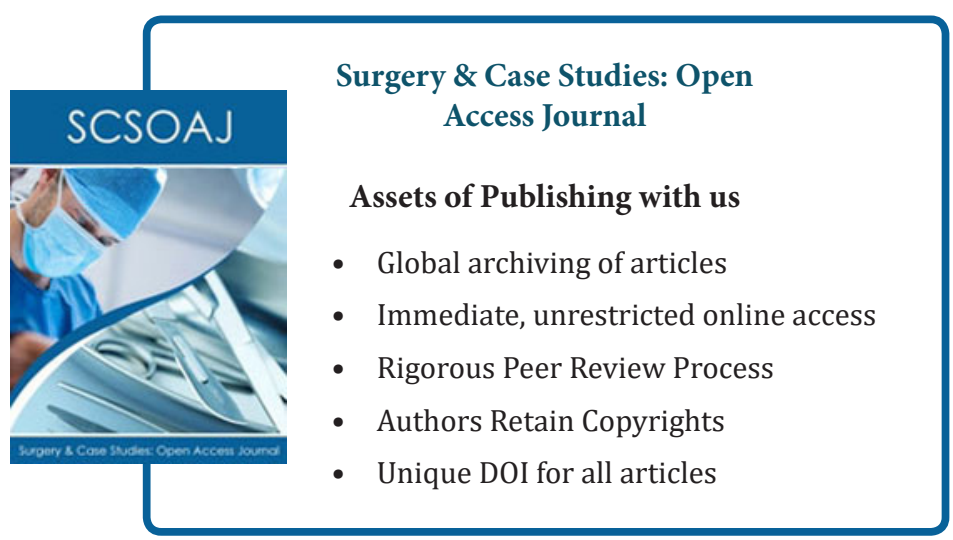

Citation: Renato Carvalho Vilella. Pulley Injury in Climbing - A New Concept to Understand and Prevent It. Sur Cas Stud Op Acc J. 2(5)- 